\title{
La situation des enfants dans le monde : la contribution dérisoire du Canada à l'aide internationale
}

1

la veille d'un autre sommet du G8 qui tentera de nouveau de concentrer l'attention des pays riches sur les problèmes de santé et de développement des pauvres du monde, il est difficile de ne pas remarquer le gouffre qui sépare le programme politique du Canada - axé sur les intérêts de 32 millions de personnes chez lesquelles la suralimentation est courante et la faim, rare - et le programme international d'organismes comme l'UNICEF, axé sur le sort d'un milliard d'enfants qui vivent et meurent dans la pauvreté, la plupart dans des familles gagnant moins de 1 \$US par jour — «la pauvreté à un dollar par jour».

Les historiens de demain décriront sûrement comme une période de stagnation la période actuelle de la politique canadienne marquée par la rancœur. Ils signaleront la paralysie du Parlement causée par le scandale, son incohérence au sujet de Kyoto, le registre des armes à feu, les dépenses militaires et l'immigration, les divisions soulevées par la définition du mariage et les promesses inépuisables d'atténuer l'insatisfaction du pays face aux soins de santé (dans un pays qui se classe déjà parmi les cinq ou six premiers par les dépenses consacrées à la santé et aux soins de santé). Entre-temps, dans le climat d'effervescence narcissique qu'est celui de la politique intérieure, on passe sous silence un scandale différent : notre gouvernement n'a pas tenu ses promesses sur le plan de l'aide internationale. Nous sommes toujours loin de contribuer, comme nous nous étions engagés à le faire, $0,7 \% \mathrm{du}$ PNB en aide publique au développement. Même le récent budget de 2005 et les 3,4 milliards de dollars canadiens supplémentaires promis en cinq ans ne nous rachèteront pas, car ce montant suivra à peine la croissance inflationniste du PNB.

Dans son rapport de 2005 sur La situation des enfants dans le monde ${ }^{1}$, l'UNICEF présente aussi, malheureusement, un bilan de stagnation. Dans une grande partie du monde, l'enfance demeure «un mot vide et une promesse non tenue». Les objectifs de développement du millénaire qui ont trait à l'enfance demeurent «sérieusement en retard» dans les catégories de l'inégalité entre les sexes (121 millions d'enfants, surtout de sexe féminin, ne fréquentent pas l'école), de la survie infantile jusqu'à cinq ans (10,6 millions d'enfants meurent chaque année de maladies évitables, dont beaucoup sont évitables par la vaccination) et des familles et des femmes (500 000 femmes meurent en couches et 15 millions sont victimes de blessures, d'infections et d'incapacités au cours de la grossesse ou suite à l'accouchement; plus de 2 millions d'enfants de moins de 15 ans sont infectés par VIH ou ont le sida).

Les experts reconnaissent qu'il serait possible de sauver l'enfance chez un grand nombre des pauvres du monde si les pays nantis contribuaient davantage à satisfaire aux besoins fondamentaux - nourriture, logement, éducation et soins de santé minimaux. Pourquoi donc le Canada se classe-t-il au $13^{\mathrm{e}}$ rang seulement sur les 22 pays de l'OCDE en terme de pourcentage du PNB consacré à l'aide étrangère officielle? Comme les dons actuels en aide s'établissent à $0,28 \%$, il suffirait de 2,3\% seulement de plus du budget fédéral (en moyenne $134 \$$ par habitant) pour atteindre l'objectif international établi par la Commission Pearson en 1969.

Réfléchissant aux causes profondes de notre échec collectif, James Maskalyk, ancien boursier en rédaction médicale du FAMC, a formulé récemment des commentaires sur le sort d'une jeune fille de 14 ans traitée dans une clinique du Zimbabwe pour un herpes disséminé secondaire au VIH. Sa grandmère s'occupait d'elle, car ses parents étaient morts du sida. (En fait, le VIH-sida a fait 15 millions d'orphelins de moins de 18 ans.) Il écrit ceci (voir $D^{r}$ Blog à l'adresse www.jamc.ca) :

«Où sont tous les autres? Pourquoi ne sommes-nous pas tous là-bas?» Je connais toutefois les réponses à cette question : notre capacité d'empathie chute de façon logarithmique en fonction de l'éloignement géographique, le taux de cette chute étant multiplié par le nombre de degrés de séparation qui existe entre nous et le problème. Cet éloignement algébrique nous portent à tourner nos réflexions vers nos propres problèmes, plus pressants non pas à cause de leur poids, mais à cause de leur proximité.

C'est bien entendu la cause de notre désintéressement, de notre négligence et de notre concentration intense sur ce que nous pouvons voir - nous-mêmes. Ne serait-il toutefois pas merveilleux que nos parlementaires prennent simplement un seul engagement sérieux et non partisan, c'est-à-dire qu'ensemble, sans égard au moment où se tiendront les prochaines élections, ni à la rancœur dans laquelle elles baigneront, ils et elles adoptent au cours de la prochaine séance du Parlement une mesure législative engageant le gouvernement et la population du Canada à porter notre budget de l'aide au développement à $0,56 \%$ de notre revenu national brut d'ici à 2010 et à $0,7 \%$ d'ici à 2015 (objectif établi récemment par la Commission européenne) $)^{2}$. Le montant de l'argent frais ou redéployé est tout à fait insignifiant par rapport à notre richesse collective. Nous devrions adopter ces objectifs, les annoncer au cours du sommet du $\mathrm{G} 8$ et les atteindre ensuite. $-7 A M C$

\section{Références}

1. UNICEF. L'enfance en péril. La situation des enfants dans le monde 2005. Disponible à l'adresse : www.unicef.org/french/sowc05/index.html (consulté le 10 juin 2005).

2. La Commission européenne. La Commission européenne se félicite de la décision du Conseil de fixer de nouvelles cibles ambitieuses pour l'aide au développement [communiqué de presse]. Document IP/05/598. 2005 mai 24. Disponible à l'adresse : http://europa.eu.int/rapid/pressReleasesAction.do?reference $=\mathrm{IP} / 05 / 598 \&$ format $=$ HTML\&aged $=0 \&$ language $=F R \&$ guiLanguage $=e n($ con sulté le 10 juin 2005). 